\title{
Sleep Consolidates Motor Learning of Complex Movement Sequences in Mice
}

Hirotaka Nagai $^{1,2}$, Luisa De Vivo ${ }^{2}$, Michele Bellesi ${ }^{2}$, Maria F Ghilardi ${ }^{3}$, Giulio Tononi ${ }^{2}$, Chiara Cirelli ${ }^{2}$

${ }^{I}$ Pharmacology, Kobe University, Japan, ${ }^{2}$ Psychiatry, University of Wisconsin-Madison, USA, ${ }^{3}$ Physiology and Pharmacology, City University of New York Medical School, USA

\section{INTRODUCTION:}

Sleep-dependent consolidation of motor learning has been extensively studied in humans, but it remains unclear why some, but not all, learned skills benefit from sleep.

AIMS AND METHODS:

Here, we compared 2 different motor tasks, both requiring the mice to run on an accelerating device. In the rotarod task, mice learn to maintain balance while running on a small rod, while in the complex wheel task, mice run on an accelerating wheel with an irregular rung pattern.

RESULTS:

In the rotarod task, performance improved to the same extent after sleep or after sleep deprivation (SD). Overall, using 7 different experimental protocols (41 sleep deprived mice, 26 sleeping controls), we found large interindividual differences in the learning and consolidation of the rotarod task, but sleep before/after training did not account for this variability. By contrast, using the complex wheel, we found that sleep after training, relative to SD, led to better performance from the beginning of the retest session, and longer sleep was correlated with greater subsequent performance. As in humans, the effects of sleep showed large interindividual variability and varied between fast and slow learners, with sleep favoring the preservation of learned skills in fast learners and leading to a net offline gain in the performance in slow learners. Using Fos expression as a proxy for neuronal activation, we also found that complex wheel training engaged motor cortex and hippocampus more than the rotarod training.

CONCLUSIONS:

Sleep specifically consolidates a motor skill that requires complex movement sequences and strongly engages both motor cortex and hippocampus. 